\title{
7. A MODEL OF INTEGRALITY OF MUSICAL AND TEACHING ACTIVITIES AT THE LESSON OF MUSICAL EDUCATION
}

Marina Caliga ${ }^{73}$

\begin{abstract}
In this article the author investigates and applies accumulated experience in music education through the methodological integrality of musical didactic activities at musical education lesson. In this regard, musical didactic activities are not only pedagogical acts of transmission of musical knowledge, but their mission is the living of sound messages, the discovery of self through art and student integration through musical didactic activities. Integrating through musical didactic activities,students discover, create, analyze, summarize, compare, apply, reflect, operating various mechanisms of musical didactic activities, becoming not only the receiver, but also the subject of musical works.
\end{abstract}

Key words: musical eduaction lesson, musical didactic activities, integrality, methodological example

Abbreviations: IMMTA - integrality model of musical and teaching activities; MTA musical and teaching activities.

\section{Introduction}

Over several millennia, man creates his own history, his own culture - a ,second nature', is the creator of a new space by knowing and changing the world. „Human knowledge has presently become a very tempting research question and at the same time it is up-to-date because one will be able to improve and enhance virtues only through a better knowledge," says I.Negură ([5, Apud: p. 9]). Following these claims, Professor I.Gagim maintains, "Man, as being integral and inclusive, the inner man (man's intimate universe) as the essence of the human being, becomes the main object of research of all areas of knowledge" [6, p. 3]. Likewise, man knows himself in the process of world cognition and change, over the millennia. This activity is based simultaneously on emotional perception and rationality: a tendency towards rationalism, awareness of emotional experience.

Being one of the forms of emotional consciousness integration, art plays an important role in spiritual activity. Man wishes to unlock the mystery of homo sapiens, hence, the human thirst for knowledge and self-knowledge, the willingness to find out the novelties as well as the secrets of the past. This enormous thirst for knowledge is the source of all human achievements that distinguishes man from other creatures existing on earth. Reflections on man and on his need for knowledge, embodied in artistic creation, are an indispensable part of philosophical and didactic analysis.

Approaching personality development education and training systemically calls for a holistic view, an integration of the education system and process, i.e. an understanding of education as a whole. To get an understanding of the totality and complexity of the educational phenomenon, this vision requires a

\footnotetext{
${ }^{73}$ Lecturer, Doctoral Candidate, „Alecu Russo” State University from Bălţi, Republic of Moldavia, email: caligamarina@inbox.ru
} 
transdisciplinary approach to the content of education and its development based on the pedagogical completeness of the educational process, through all forms of education (formal, non-formal, informal) and scopes of education (intellectual, moral, aesthetic, etc.) engaged in pursuing the same education ideal, with the involvement in this education process of all stakeholders (family, school, society, church).

\section{Research Problem}

As result of curricular reforms in Moldova (from 1997 to 2003, 2004, 2010), the musical education discipline was framed as a distinct and autonomous educational field in educational practice. The systematized teaching approach of musical activities at the musical education lesson has been implemented in practice for more than two decades, however, there hasn't been carried an insightful and conceptualized research on the concept of integrality of musical teaching activities at the lesson of music. From the perspective of integrality, musical teaching activities triggered a long process of reconceptualization of discipline, reconstruction and development of curriculum, reassessment and restructuring of its content, reconsideration and adaptation of methodology entirety of musical didactic activities to the principles of art.

Curriculum reform involved a substantial change in the educational process, a new model of teaching, and namely integrated teaching of this discipline, has been proposed allowing the students to form a unified concept about music. With this idea in mind, it was necessary to carry research and implement the concept of integrated musical activities, in which the students are directed to achieve, to integrate their ideas with those of their colleagues, to evaluate their work and issue assumptions and conclusions that support their arguments and to find application etc. As it has been stated, we know about the musical education conceptual framework, the Curriculum, guides, manuals, content, technologies and methodologies etc., creates the vision that it is sufficient for musical education inegrality by means of musical and teaching activities. From the perspective of modern principles, this does not exclude the need of reconceptualizing the music lesson approach to achieve integrality by means of musical and teaching activities.

Reconceptualization provides applying modern methodology as well as benefiting from students' interests and learning motivations by various musical and didactic activities at the lesson. In this respect, the lesson integrality by musical and didactic activities becomes a cognitive and emotional constituent of musical proficiency at the dynamic and integral process level. Thus, knowledge and students' skills and attitudes integrate, sistematising them via musical and didactic activities and dynamic processes through areas: cognitive, psychomotor and attitude skills and turned into music - the main outcome of music education.

A real change in the didactics of musical education has been produced and developed by experienced researchers and professors in the Republic of Moldova, to name but a few: A. Popov [11],T. Bularga [2], M. Vacarciuc [13], I. Gagim [6], M. Morari [8], Vl. Babii [1], L. Graneţkaia [7], M. Cosumov [3], V. Crisciuc [4], the research problem being investigated using methods and 
procedures, techniques and principles of integrating knowledge, skills and attitudes by means of musical and didactic activities, thus consolidating various aspects of the specifics of musical art. While mentioned above researchers' efforts are valuable, as they set the fundamentals in many directions in the field, the issue of specific methodology for musical and didactic activities integration has not been solved. This is because it has been approached as a didactic issue, and not as a defining action of integration of the individual in society. In this respect, AMD integrality is interpreted differently either because of the association with some instructional methods and principles already promoted, or correlation to different theories, systems, experiences etc.

A wide opening to practical solutions in the methodology approach to musical education lesson and content integrated by musical and didactic activities has been offered by one of the founders of curricular reform for the discipline "music education in the contemporary school is built on a system of regularities and principles that assigns value to educational and scientific entities" [6, p. 10]. Analyzing world training / development systems of music lessons from the evaluative perspective I. Gagim concludes "Musicology represents music epistemology; it leverages modern definitions of the main categories of music in each of the four main forms of musical activity: creation, interpretation, hearing and analysis - which become forms of musical experience, different in content, yet integrated via the act of communicating with music "[6, p. 3].

Developing the same idea, M. Morari affirms: "By means of initiation forms of music one achieves going deep into the world music; concepts, categories, theoretical definitions being learned as a result of musical act [8, p.20]. According to researcher I. Gagim, " the system of students' musical-didactic activities is produced by derivation of the four forms of musical activity creation-interpretation-listening-analysis. Each of these forms of musical and didactic activity has an integrating and multifunctional character of learning activities, educational technology, initiation into the mysteries of music, of musical-artistic and musical-pedagogical experiences; musical, artistic and pedagogical experiences have double function: sub-system of a music lesson system and autonomous system of operating / assimilating the value of music " [8, p.11].

From a practical standpoint, I. Gagim investigates musical didactic activities attributing value to each of the musical teaching activities, motivating their status as "learning activities" [6, p.43]. "Thus, in musical general education the model of integrated teaching is an update of studying music under the creative principles, which corresponds to formative education, designating both a coherent assembly of content, teaching/learning and assessment methods, as well as a system of decision making processes, of monitoring the entire educational process " [12, p. 61]. In the course of this research we advocate a theoretical and practical model of integrating musical and didactic activities as a systemic approach to musical educational process at the music lesson. 
Research of practice under this approach demonstrates, however, that this guidance is not sufficient to achieve the aims of the music education in school. Having mentioned paper, Professor I. Gagim concludes by "re" - definining music education: "the concept of musical education must employ scientific instrumentation advancement of the educational process: from formation of musical culture to defining musical culture within the context of students' spiritual culture" [6, p. 18]. Analyzing M.Morari's statements, we may emphasize: "Conceptualization and realization of the Curriculum in music education is based on the specific nature of musical art and music reception by students. Review of Moldovan music education content in school first, and then development of music education Concept and music education Curriculum constituted the new orientation of the school discipline - music education" [8].

Thus, the modern curriculum stipulates training students' musical skills, requesting teachers to use a varied theoretical praxiological approach, systematized and integrated through updated educational processes. Music education has gone through a profound process of reconceptualization, adapted to methodological principles of musical and teaching activities at the lesson. In addition, we emphasise the need for a qualitative correlation of theory and practice in order to obtain performance at the music education lesson.

\section{A model of integrality of music-teaching activities at the lesson of musical education}

Integrality of of music-teaching activities in music education class is not just a teaching act of transmitting music knowledge or knowledge about music but also a profound music experience, detection of the sound message, discovery and investigation of the truth. In this lively and dynamic process during the lesson the student is not only the receiver but also the authenticated subject of the the musical work. The main character, the student is the one who discovers, creates truths, shows attitude, operating the mechanism of musical knowledge through musical and teaching activities. In this regard, the integrality model of music-teaching acticities at the lesson will contribute to: student's comprehension, analysis, synthesis, comparison, characterization, generalization and application of this dynamic and integral process.

The theoretical foundation of Integrality Model of Music-Teaching Activities (IMMTA) was focused on:

- The integration of theoretical and practical operational IMMTA to form students' attitude to the lesson of music;

- Architectural construction of the lesson of music after MTA concept [6, p.45];

- IMMTA from the music subject to the vivid perception of the lesson message;

- IMMTA after the principle of internalizing the music as the basic integrating principle in music education $[6$, p. 9; p. 11]; [5, p. 59];

- Process and dynamic systematising MTA from primary perception to inner experience;

- Students' integration through the systemic integrallist mechanism of MTA; 
- IMMTA through cognitive-affective technology;

- IMMTA after the four directions of one and the same arch - of knowledge: learning to know, learning to do, learning to live together, learning to exist [9];

- Training students' attitude at the music lesson in relation to IMMTA [10, p. 30-31].

The model consists of parts, identifiable with school curriculum components in Moldova - MTA epistemology, content, methodology. Epistemically motivated by a conceptual basis, MTA epistemology integrates topics with systematized procedural dynamic actions at the music lesson. IMMTA components are integrated and systematised by sentence in relation to music education curriculum structure in Moldova.

MTA epistemology is the ideas, concepts, principles, theories on musicexisting educational systems and their integration into the music lesson. Detailed description of the systemic integrality concepts and their application in educational processes (philosophy, pedagogy, psychology, musicology); research of the concept of transdisciplinary - as an integrating factor: the four directions of one and the same arch - of knowledge: learning to know, learning to do, learning to live together, learning to exist, aiming at: learning to change yourself and transform society.

A systemic approach to music education process: retrospective and insight analysis of MTA in different schools and periods; concept of music-teaching activity; music lesson concept - micro structural components and their integration via MTA, architectural construction of the music lesson; attitude concept towards MTA; MTA methodological principles of integration: IMMTA from theory to practice and vice versa, from practice to music education theory, internalization and externalization through IMMTA, procedural-dynamic systematisation of MTA, continuity and gradualness of MTA complexity; classification and structuring IMMTA principles, methods, techniques and forms at the music lesson.

MTA contents apply via the musical teaching material (pieces of music, different teaching materials based on pupils' MTA). The contents are the main source of the MTA, their approach / integration into the music lesson is represented by:

- Principles (concepts, ideas) established via MTA and focused on taching and training activitaty;

- Operational objectives, IMMTA teaching tasks;

- Music and music-didactic theory through which MTA will be integrated;

- Methods, processes, techniques, forms and means for MTA.

IMMTA methodology is built on several criteria:

- Triadic construction of the concept of internalization / externalization of music [I.Gagim];

- Integrality of content teaching through cognitive and affective aspects;

- Putting into use the route of perception process of IMMTA systematization by: primary perception and inner experience; 
- Integrality of musical education lesson in relation to operational objectives taxonomy;

- Students' attitude toward IMMTA at the lesson of music education.

The proposed methodology addresses the instructive, educational and training issues of the students' attitude towards IMMTA at the lesson of musical education and integrated testing at the level of knowledge, skills, attitudes. In the theoretical part of our research it has been proven that all musical activities within a lesson, united under one goal, intertwining and completing each other, follow one direction - learning the theme of the lesson, approaching it differently (audit, interpretative, theoretical and analytical, creative, etc.), different aspects of understanding the musical phenomenon called into question (otherwise, any topic is nothing but a musical phenomenon, a fundamental aspect, a law of musical art). Besides the mentioned aspects, my intention was to analyze the presence (or absence) of the experience of communicating with the musical art and students' awareness of the need to communicate with music via IMMTA at the lesson of musical education. The Figure Nr. 1 describes the theoretical and practical experiment to achieve the scope of the problem under study regarding integrality of the music education lesson via music-teaching activities.

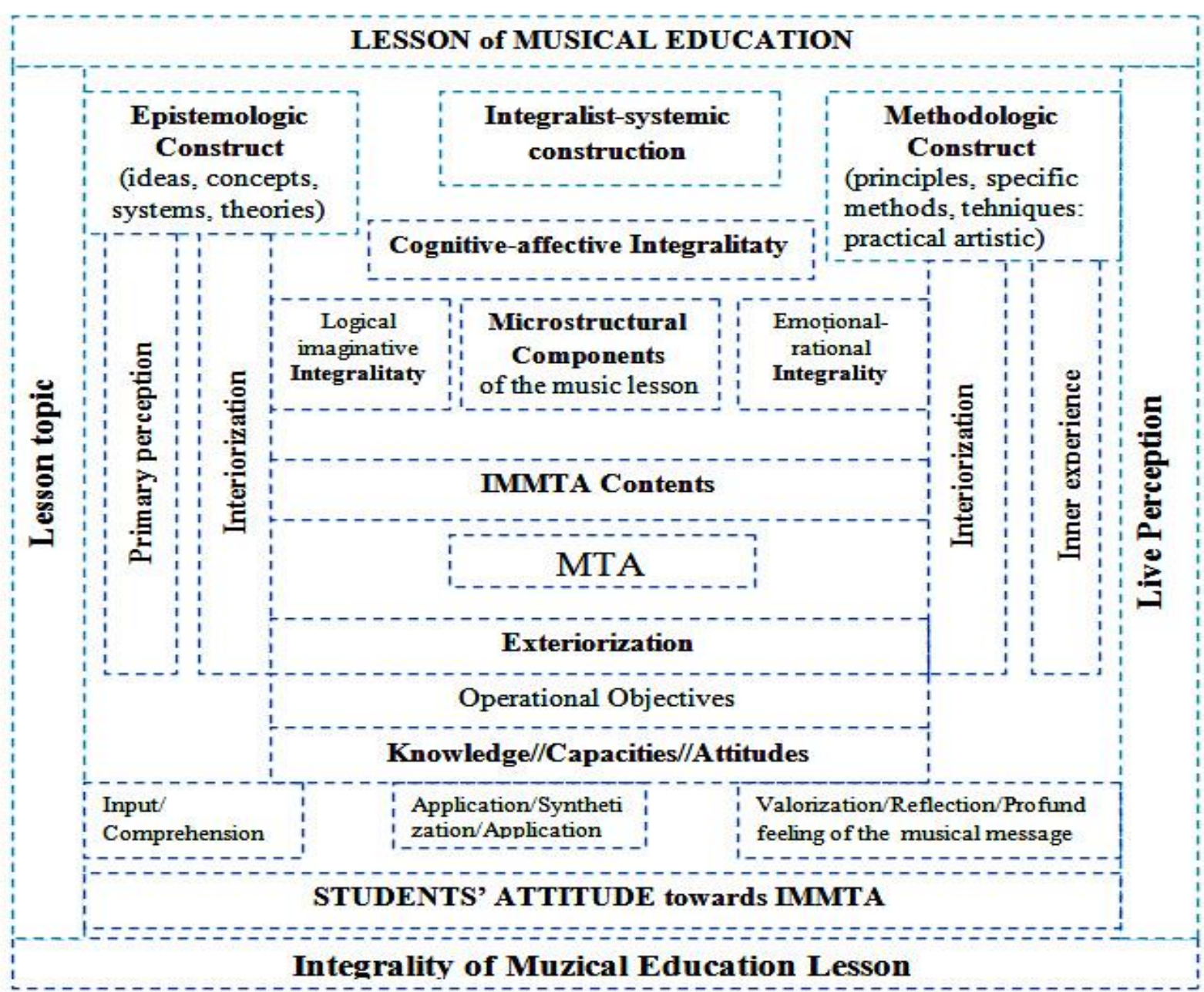

Figure Nr. 1 The model of integrality of musical and teaching activities at the lesson of musical education 


\section{Conclusions}

The evolution of the recent decades allows to identify patterns that contributed to the development of music education didactics. Thus, Music Education is a pedagogical discipline at the crossroads between art and music, pedagogy and psychology, philosophy and musicology etc. Reference elements for the development and expansion of the educational process of ME have emerged, over time, with more directions, focusing on concepts, specific methods, different by their complexity, viewed differently from one educational system to another. Researchers from various scientific fields (pedagogy, musicology, sociology, economics, philosophy, psychology, etc.) carry an inventory of pedagogical ideas and values of the phenomenon under study. Conditioned by educational interests of society, the idea of educational integrality is put forward and requires theoretical and practical foundation.

The integrality issue concerns many reference areas, arising from current guidelines of education by developing new approaches to integration. These guidelines, interests and developing visions, from a postmodern perspective, require research of integrality in music education as a general term, as well as in the narrow sense, specific to this field. Accepting the integrality character, the complexity and the progressive changes of training / education actions both by the student and by the teacher, will consciously lead to setting a new quality of building the paradigm of long-term correlation pedagogical phenomena, forming a realistic vision of the dynamics of the overall educational integrality development: teacher - student; student - teacher. The current understanding of education is generated by concepts, principles and areas clearly delineated and prioritized, set in motion by properly formulated rationality.

Analytical treatment of the concept evolved in the context of actions that produce numerous strategic behavior, puts the basis for a whole system of re / conceptualizations of musical education. Within the context of our research, training student's personality by cognitive-affective integrality at the lesson of musical education is classified by mental processes: cognitive-affective and psychomotor (mentioned in the previous subchapter). According to the mentioned above researcher s, who have investigated the personality formation via cognitive and affective aspect, both aspects are equally important in logicalartistic integrality. Following this finding, we observe phases-stages of communication in music education lesson integratng perceptive processes: primary perception - a permanent dialogue with / through musical and teaching activities from teacher to pupils and vice versa; the transition from inside to outside in the perception of music-teaching activities; integration / transformation of physical matter into spiritual matter; interiorization of musical message as an integral process. 


\section{References}

1.Babii, Vl. (2005). Efficiency of Musical-Artistic Education, Chişinău: ElenaVI Publishing House

2. Bularga, T. (2008). Psycho Pedagogy of Interest towards Music. Bălţi: Bălţi University Press

3 Cosumov, M. (2012). Homework in Musical Education as an Element of Curriculum. [PhD thesis]. ped. Chişinău

4. Crisciuc, V. (2013). Specifics of teaching music knowledge (within Music Education discipline). [PhD thesis]. ped. Chişinău

5. Gagim, I. (2003). The Psychological Aspect of Music. Iassi: Timpul

6. Gagim, I. (2004). Psyhhopedagogical and Musicological Fundamentals of Musical Education. [Scientific review of the Doctor Habilitate Thesis based on works published in pedagogy]. Chişinău

7. Graneţkaia, L. (2008). Didactic Tehnologies of Interpretative Analysis of Image in Music in the Process of Piano Training of Teachers of Music. $[\mathrm{PhD}$ thesis]. ped. Bălţi

8. Morari, M. (2005). Evaluating Students' Musical Culture in the Process of Realizing School Curriculum. [PhD thesis]. ped. Chişinău

9. Nicolescu, B. (2010). Transdisciplinarity. Manifest. Iaşi: Junimea

10. Pâslaru, Vl. (2013). Introduction to the Theory of Literary and Artistic Education, (second ed.). Bucureşti: Sigma

11. Popov, A. (1993). Differentiated Instruction in the Process of Developing' Melodic and Rhythmic Hearing Lower School Age Students. [PhD thesis]. ped. Chişinău

12. Stici, A., Gagim I. (20013). Methodology of Musical Education. For ShortTerm Higher Education Institutions Students Specialized in Music and Teaching Music. Chişinău

13. Vacarciuc, M. (2002). Spiritual Development of Young Learner via Music and Singing. Methodologic Guide. Chişinău: Ştiinţa 